\title{
Modeling Partial Discharge in a Three-phase Cable Joint Experiment with Minimal Adjustable Parameters
}

\author{
G. Callender, P. L. Lewin \\ The Tony Davies High Voltage Laboratory \\ School of Electronics and Computer Science \\ University of Southampton \\ Southampton, SO17 1BJ, UK \\ J. A. Hunter \\ Mott MacDonald \\ Brighton, BN1 4FY, UK \\ and $\mathbf{P}$. Rapisarda \\ Vision, Learning and Control Group \\ School of Electronics and Computer Science \\ University of Southampton \\ Southampton, SO17 1BJ, UK
}

\begin{abstract}
A general physical model of partial discharge (PD) has been developed and used to simulate discharges within a void at the tip of a metallic spike defect within a three-phase $11 \mathrm{kV}$ paper insulated lead covered (PILC) cable joint. Discharges are modeled by altering surface charge density at the void boundary using a logistic function distribution. The model was validated against experimental data, and a good agreement was observed with minimal free parameters. The model was then used to investigate the impact of single phase energization on PD activity in three-phase PILC cable joints. It was concluded that PD testing of three-phase PILC cable joints should be performed at raised temperatures with the cable fully energized as this results in a higher frequency of PD activity, and reduces the level of background PD from cable terminations. This research represents a further step towards developing PD models that can describe measurements taken from operational high voltage plant.
\end{abstract}

Index Terms - Partial discharges, cable insulation, finite element methods.

\section{INTRODUCTION}

PARTIAL discharges (PDs) are defined as "a highly localized or confined electrical discharge within an insulating medium between two conductors" in [1]. Due to the high dielectric breakdown strength of insulating materials, PDs typically occur within defects in the insulation. Consequently, measuring PD activity is now one of the main prognostic techniques in determining the health of high voltage plant [2]. To gain a more complete understanding of the physics of PD activity numerous experiments and simulations have been performed. These typically investigate PD activity from an artificial defect, typically a spherical or cylindrical void, surrounded by insulation material between parallel plate electrodes under alternating current (AC) conditions [3-5]. In this arrangement, the applied electric field is a linear sinusoid, and the commonly observed "rabbit ear" phase resolved partial discharge (PRPD) patterns are well described by existing PD models [6,7].
Unfortunately close agreement between simulation and measurements does not extend to the complex PRPD patterns observed from data taken on-site from operational plant, where multiple sources and high levels of background noise can make a physical interpretation of observed data exceptionally difficult [8]. Three-phase cable experiments provide a middle ground between on-site measurements and highly controlled parallel plate experiments [9]. In these experiments artificial defects, designed to replicate real world faults, were investigated in three-phase cable sections [10]. Despite the complex nature of the PD data generated in these experiments, a physical PD model was found to be able to replicate the experimental data for certain cases. It should be noted that the model requires knowledge of the defect type and scale, information not generally available on-site.

In this work a model has been presented that simulates PD activity from an experiment where an artificial metallic spike defect is placed on the ferrule of a phase inside a paper insulated lead covered (PILC) three-phase cable joint. A cable joint was 
investigated because this component is usually fabricated on-site, often in challenging conditions, so may be more prone to flaws from human error [9]. The spike defect is designed to reproduce the effects of poor solder smoothing during joint construction. Based on the experimental observations it is assumed that PD activity occurs inside a void at the tip of the spike, which means the system's geometry is a significant departure from a parallel plate arrangement. The model uses finite element software to solve a three-dimensional electrostatic equation, and models PD activity by altering the surface charge density at the void boundary, a technique that has been used previously [11]. The model expands the earlier work in two important ways. Firstly, the surface charge density is a continuous function, which removes numerical stability issues associated with a discrete function used in earlier work [11]. The second improvement is that PD activity is governed by the electric field at a number of points in the defect, rather than the electric field at a single point, typically the center for a spherical void. The experiments and simulations show reasonable agreement despite the simplifications used. The model is then used to investigate the possible impact of different phase energization on PD activity.

\section{CABLE JOINT CONSTRUCTION}

To fabricate the sample a straight lead joint was introduced into a three-phase PILC cable section using the same methods employed on-site. A straight lead joint was constructed as they are the most commonly used joint type for three-phase PILC cable circuits [9]. The first step in constructing a straight lead joint is to cut away the cable over sheath and armor to the required length. The layer of bitumen-impregnated hessian beneath is removed with a knife and the bitumen is then cleaned with a cloth and some solvent. At each end of exposed lead the armor wires are bonded to the sheath by soldering with a tin and lead alloy, which is then wrapped with electrical tape which acts as a layer of mechanical protection. The rest of the lead sheath is then removed to expose the paper belt which is discarded to allow access to the paper insulated phase conductors and bedding. At the location where the phase conductors overlap the paper insulation is removed to provide access to the copper conductors. The remaining paper insulation is covered in cotton paper saturated in oil to protect the paper insulation during the ferrule soldering process, as shown in Figure 1. Ferrules are used to connect the copper phases and then molten solder is repeatedly applied to create a solid connection, this technique is known as "sweating."

Once the solder has cooled, the ferrules are sanded to remove spikes in the solder which could enhance the electric field leading to increased electrical stress on the insulation. The cotton paper is then removed and oil impregnated Crêpe paper is wound several times around each phase to replace the previously removed insulation. A lead capsule is then fabricated to surround the joint and soldered to the lead sheath of the cable at each end. The lead capsule has two holes cut into the top and then hot liquid bitumen is poured through one of these holes with the other left free to allow air out. After the bitumen has cooled the holes are sealed, which completes joint construction.

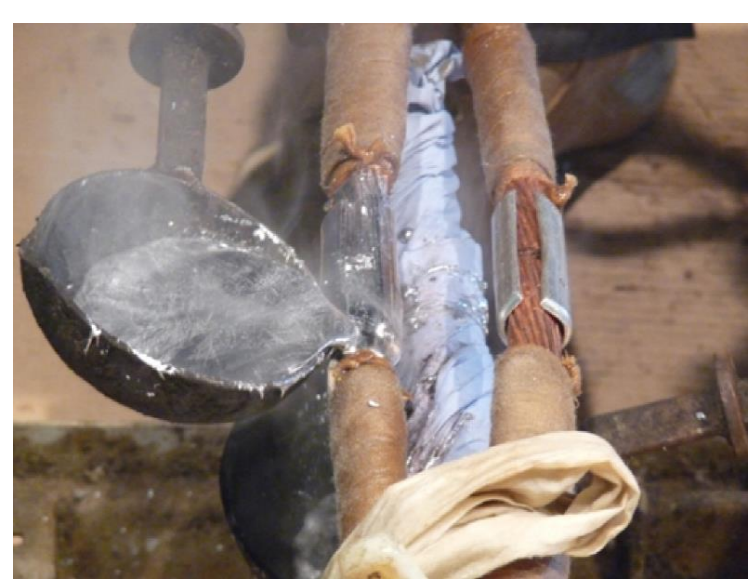

Figure 1. Photograph showing the application of molten solder to a ferrule in a straight lead joint, used with permission from [9].

\section{PARTIAL DISCHARGE EXPERIMENT}

The experiment was designed to replicate the effects of poor solder smoothing on the ferrule during joint construction. During the fabrication of the joint used in the experiment a metal spike was created by applying solder to a wire, which was attached to the ferrule on phase two in the direction of phase one. The geometry of the arrangement is shown in Figure 4. The spike tore through the innermost layers of Crêpe paper when it was wrapped around the ferrule, but was fully surrounded by paper when the insulation application was complete. Due to the time and cost constraints of the experiment, the spike was $14 \mathrm{~mm}$ in length so that PD activity occurs as soon as the experiment begins [9]. The spike defect is therefore more extreme than real world cases, but does not create sufficient damage to cause an immediate breakdown. A schematic of the experimental set up is provided in Figure 2.

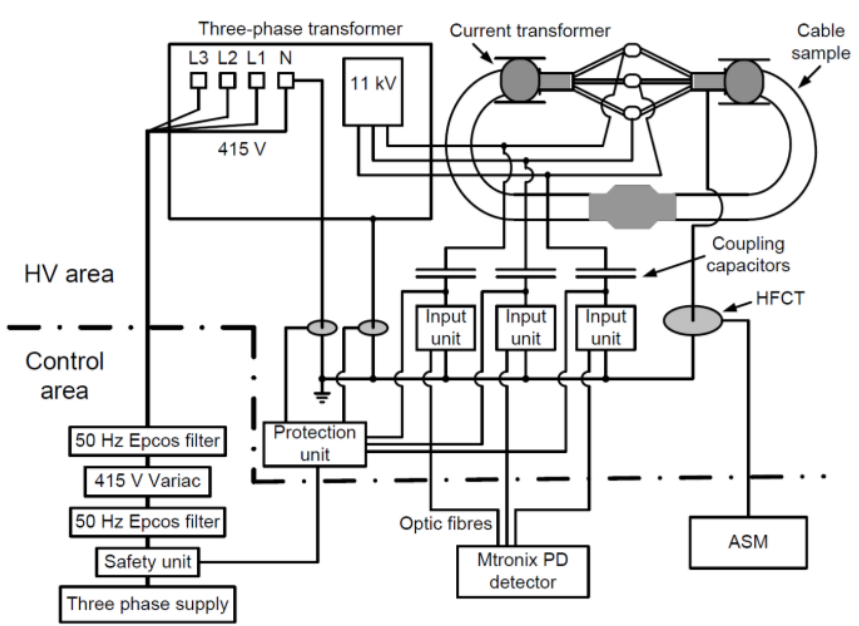

Figure 2. Schematic diagram of PD measurement system, used with permission from $[9,10,12]$. The cable sample is $10 \mathrm{~m}$ and contains a threephase straight lead joint with a spike defect on phase 2 .

PD activity is measured on each phase using Omicron's Mtronix MPD600 PD measurement equipment. The input unit from the measuring equipment was connected in series with $1 \mathrm{nF}$ blocking capacitor on each cable phase. The entire 
experiment was performed at rated volts which corresponds to $11 \mathrm{kV}$ root mean square (RMS) line to line voltage at $50 \mathrm{~Hz}$.

Initially the conductor temperature of the joint was held at $16{ }^{\circ} \mathrm{C}$ and no PD activity distinct from the background noise from cable terminations was measured [12]. The conductor temperature was then raised to $65^{\circ} \mathrm{C}$ and $\mathrm{PD}$ measurements taken as the sample cooled. The conductor temperature was determined by measuring the surface temperature of the joint and using thermal calibration data [12]. At higher temperatures PD activity distinct from the background noise was observed. Two PD data sets, in the following called data set 1 and data set 2 , collected over $19.8 \mathrm{~s}$ and $6.4 \mathrm{~s}$ respectively were taken from phase two when the conductor temperature was $56{ }^{\circ} \mathrm{C}$. PD activity from the spike occurs over a narrow range of phase angle, $\psi$, from approximately $80^{\circ}$ to $165^{\circ}$, with a peak apparent charge, $q_{\mathrm{PD}}^{\prime}$, of $2.38 \mathrm{nC}$ and an average of 1.2 PDs per cycle. PRPD patterns are shown in Figure 3.

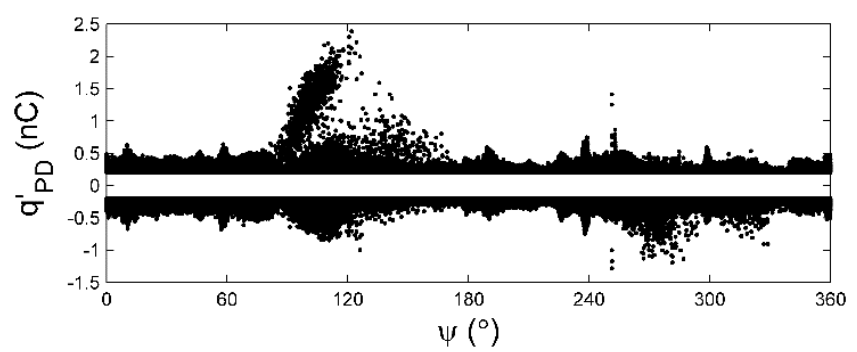

(a)

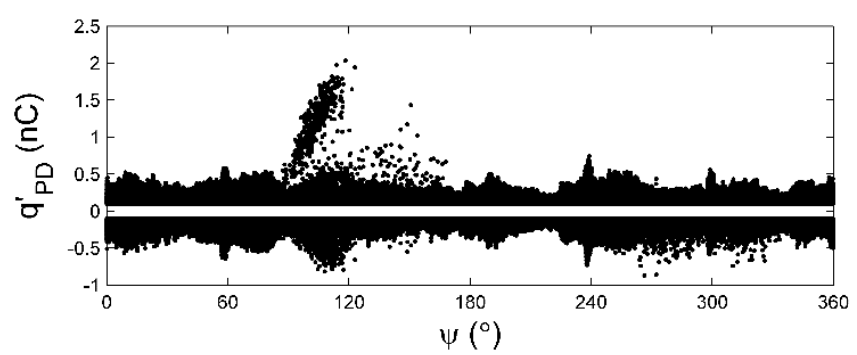

(b)

Figure 3. PRPD plots for (a) data set 1 and (b) data set 2. PDs from the spike defect can be clearly observed between $80^{\circ}$ and $165^{\circ}$.

\section{PARTIAL DISCHARGE MODEL}

In this section an overview of the model is given, including the underlying assumptions and governing equations. The fundamental simplification used in the model is that the measured discharges are taking place in a spherical void at the tip of the spike. This is because the ferrule is surrounded by oil impregnated Crêpe paper, which has a high electrical breakdown strength, so it is unlikely that the discharges are propagating through the paper. A more plausible explanation is that air was trapped around the spike due to the deformation of the paper layers by the spike. The tight wrapping of consecutive layers of paper around the spike would then force trapped air to the spike tip. As the Crêpe paper is surrounded by oil at high pressure it is reasonable to suppose that the void has deformed to be approximately spherical.

\subsection{MODEL GEOMETRY}

The model must solve an electrostatic equation within the insulation material, so the physical boundaries of the computational domain are the cable sheath and the three conductor phases with a spike on phase two as shown in Figure 4. The spike is considered as a conical frustum and a sphere, with the sphere placed such that the spike surface is smooth. The region of the spike surface created by this sphere is hereafter referred to as the spike tip. The base of the frustum is placed such that the entire bottom circle of the frustum is just inside phase 2 . The void is assumed to be spherical, with the same origin as the sphere used to define the spike tip. The domain is truncated in the axial direction of the cable joint, $3 \mathrm{~cm}$ was found to be sufficient, as the void is the region of interest for PD modeling. There are therefore 8 parameters required to

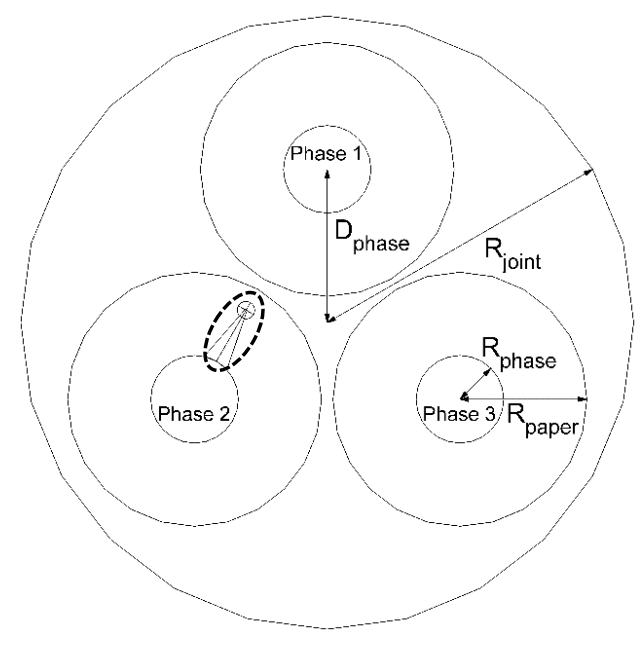

(a)

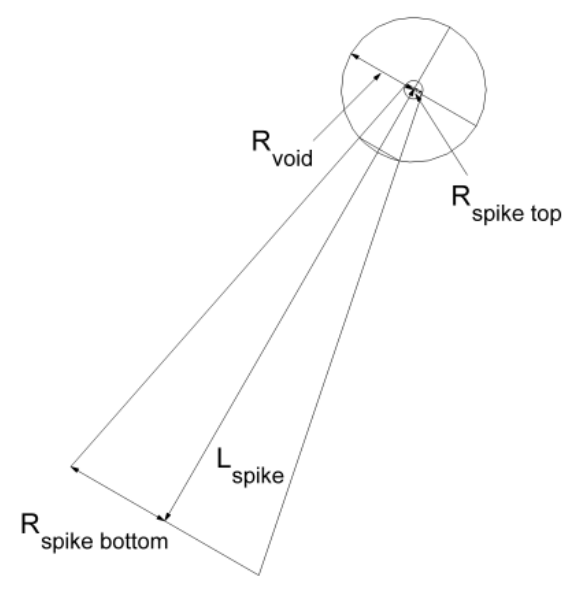

(b)

Figure 4. Figure showing the model geometry with the geometry parameters and phase conductors labeled: (a) full geometry and (b) zoom of the spike defect corresponding to the dashed region in (a).

specify the geometry: the radius of the joint $R_{\text {joint }}$, the distance of the conductors from the joint center $D_{\text {phase }}$, the radius of the 
conductors from the phase center $R_{\text {phase }}$, the radius paper region surrounding each phase $R_{\text {paper }}$, the length of the spike $L_{\text {spike }}$, the top radius of spike conical frustum $R_{\text {spike top }}$, the bottom radius of spike conical frustum $R_{\text {spike bottom, and the }}$ radius of the void $R_{\text {void }}$. All these parameters can be set by the conditions known in the experiment except the void radius, which will be adjusted to fit the data. All geometry parameters are shown in Figure 4, geometry parameter values are given in Table 1.

\subsection{DISCHARGE PHYSICS}

The standard assumption for a PD to occur within a void is that there must be a sufficiently high electric field and an available electron, these conditions will be referred to as the field condition and the free electron condition respectively [13]. The standard reasoning is that the electric field accelerates the available electron to a sufficient velocity to start an electron avalanche, beginning the discharge process. To explain the presence of PDs in only one half cycle of the applied voltage the electron generation processes must be considered. The possible sources of free electrons in the void are emission from the metallic spike in the negative AC cycle, background radiation and surface emission of charge from previous PDs. As background radiation and surface emission processes will generate electrons throughout the AC cycle, and PDs are only observed in the negative half cycle, it is assumed that free electrons for PDs must come from spike surface, which only occurs in the negative half cycle. The magnitude electric field is too low for field emission, and the temperature is too low for thermionic emission. A possible mechanism of electron generation in the conditions present in the experiment is ion bombardment.

Discharges may not always begin exactly on the symmetry axis of the spike, but experimental images of PDs from spikes show that discharges generally begin from the tip of the spike, [14]. To model this, the inception and free electron conditions will be considered at a number of points on the spike tip. The spike tip will considered to be the region of the spike created by the sphere, and the points will be called inception points. It is clear that both the field and free electron condition will be dependent on the electric field normal to the surface of the spike.

The field condition states that for a discharge to occur at a given point the electric field at that point exceeds an inception value $E_{\text {inc }}$. Discharges will propagate in the direction of the electric field at the inception point, which is normal to the spike surface. The PD will deposit charge on the void surface until the electric field at the inception point has fallen to an extinction value $E_{\text {ext }}$. The discharges propagate in the direction of the electric field during the negative half cycle, so the charge deposited on the void boundary will be negative. The effect of a single discharge is shown qualitatively in Figure 5.

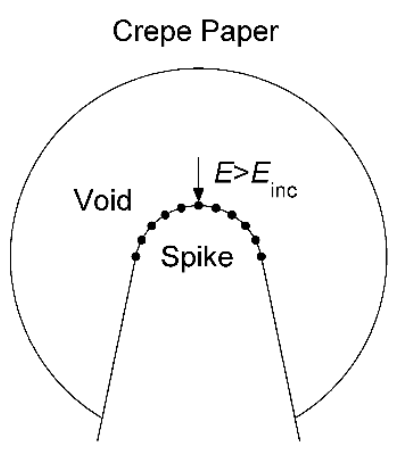

(a)

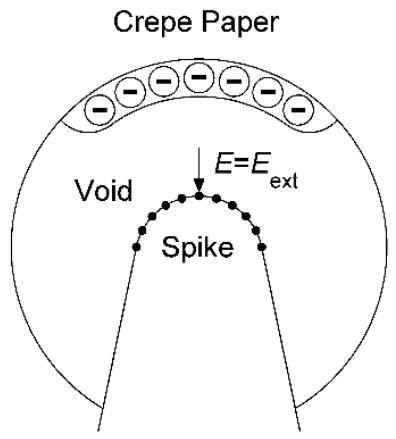

(b)

Figure 5. Figure showing qualitatively the effect of a single discharge in the void starting from an inception point in the centre of the spike tip: (a) before the discharge, (b) immediately after the discharge. The black dots represent inception points on the spike tip and $E$ is the electric field at the inception point initiating the discharge.

\subsection{ELECTRIC FIELD}

The electrostatic potential $V$ of the system is found through the solution of the electrostatic equation

$$
\nabla^{2} V(\vec{x}, t)=-\frac{\rho(\vec{x}, t)}{\varepsilon_{0} \varepsilon_{r}(\vec{x})}
$$

where $\vec{x}$ is a spatial coordinate in the model geometry, $t$ is time, $\rho$ is the volume charge density, $\varepsilon_{0}$ is the permittivity of free space and $\varepsilon_{r}$ is the relative permittivity with $\varepsilon_{r}=\varepsilon_{r \text { paper }}=$ 3.6 in the paper, $\varepsilon_{r}=\varepsilon_{r \text { oil }}=2.7$ in the oil and $\varepsilon_{r}=\varepsilon_{r \text { void }}=$ 1 inside the void. The boundary conditions are set to be

$$
\begin{aligned}
& V(\vec{x}, t) \\
& =\left\{\begin{array}{cl}
0 & \text { at joint sheath } \\
U_{0} \sin (\omega t) & \text { at phase } 1 \\
U_{0} \sin (\omega t+2 \pi / 3) & \text { at phase } 2 \text { and spike } \\
U_{0} \sin (\omega t+4 \pi / 3) & \text { at phase } 3,
\end{array}\right.
\end{aligned}
$$

where $\omega=100 \pi$ and $U_{0}=22 / \sqrt{6} \mathrm{kV}$ which corresponds to $11 \mathrm{kV}$ RMS line to line voltage with a $50 \mathrm{~Hz}$ AC supply to match the experiment. The electric field $\vec{E}$ can then be found through

$$
\vec{E}(\vec{x}, t)=-\vec{\nabla} V(\vec{x}, t) .
$$

At the axial boundaries of the domain, the following boundary condition is applied

$$
\varepsilon_{0} \varepsilon_{r}(\vec{x}) \vec{E}(\vec{x}, t) \cdot \vec{n}_{\text {axial }}(\vec{x})=0,
$$


where $\vec{n}_{\text {axial }}$ is the normal unit vector of the boundary plane in the axial direction of the joint. This boundary condition is chosen because far from the void the electric field approaches the applied field solution for a three-phase cable joint without a spike. The volume charge density $\rho$ depends on PD activity inside the void, and will be discussed in Subsection 4.4.

\subsection{SURFACE CHARGE DYNAMICS}

PDs are modeled by altering the surface charge density, $\sigma$, at the boundary of the void with the Crêpe paper. It is assumed that during the experiment charge does not move into the bulk of the insulation material. As the void is assumed to be spherical, the volume charge density $\rho$ can be written as

$$
\rho(r, \theta, \phi, t)=\delta\left(r-R_{\text {void }}\right) \sigma(\theta, \phi, t),
$$

where $\delta$ is the Dirac delta function, $R_{\text {void }}$ is the void radius and $r, \theta, \phi$ are the standard spherical polar co-ordinates with their origin at the void centre. As discharges occur in the order of nanoseconds, compared to the AC cycle period of milliseconds, the discharge event is treated as instantaneous for modeling purposes. The $i$ th discharge occurring at $t_{\mathrm{PD} i}$ adds surface charge density $\sigma_{\mathrm{PD} i}$ to the existing surface charge

$$
\sigma\left(\theta, \phi, t_{\mathrm{PD} i}^{+}\right)=\sigma\left(\theta, \phi, t_{\mathrm{PD} i}^{-}\right)+\sigma_{\mathrm{PD} i},
$$

where \pm superscripts denoted times immediately before and after respectively. To simplify the model $\sigma_{\mathrm{PD} i}$ is a charge spot defined such that the charge from any inception point spreads across the entire void surface while requiring that charge is at least a distance $\pi R_{\text {void }} / 20$ from the symmetry axis of the spike. $\sigma_{\mathrm{PD} i}$ is set to be a logistic function so

$$
\begin{aligned}
& \sigma_{\mathrm{PD} i}\left(\theta, \phi, \alpha_{i}\right) \\
& =\frac{\alpha_{i}}{1+\exp \left(\beta\left(\Theta\left(\theta, \phi, \theta_{\mathrm{PD}}, \phi_{\mathrm{PD}}\right)-19 \pi / 20\right)\right)},
\end{aligned}
$$

where $\alpha_{i}$ is negative and adjusted so that the magnitude of the electric field at the inception point undergoing PD is reduced to $E_{\text {ext }}, \beta=100 / \pi$ determines the rate of spatial decay of charge at the boundary, and $\theta_{\mathrm{PD}}=\pi / 2$ and $\phi=\pi / 3$ are the azimuthal and polar angles of the center of the charge spot. $\Theta$ is the central angle between two points on a sphere, defined as

$$
\begin{aligned}
\Theta\left(\theta_{1}, \phi_{1}, \theta_{2}, \phi_{2}\right) & =\cos ^{-1}\left(\cos \left(\theta_{1}\right) \cos \left(\theta_{2}\right)\right. \\
& +\sin \left(\theta_{1}\right) \sin \left(\theta_{2}\right) \cos \left(\mid \phi_{1}\right. \\
& \left.\left.-\phi_{2} \mid\right)\right),
\end{aligned}
$$

where $\theta_{i}$ and $\phi_{i}$ are the polar and azimuthal angles respectively of point $i$.

The physical justification for our choice of $\sigma_{\mathrm{PD} i}$ is that the data sets to be modeled are taken after sustained discharge activity, which means that the surface conductivity of the void surface will be much greater than that of the virgin Crêpe Paper. Therefore charge from a PD could feasibly spread across the entire boundary between the void with the Crêpe paper. Charge is prevented from moving too close to the spike because the electric fields at the surface of the spike will oppose the negative charge from the PD during the negative half cycle, preventing charge spreading close to the spike. The consequence of this assumption is that discharges in the model have a relatively high magnitude, because the charge spreads across the entire void surface, and a low frequency, because a discharge any inception point causes a reduction in the electric field at all inception points. This PD behavior is also observed in the experimental data, where high magnitude PDs, in the order of nanocoloumbs, are observed with an average of only 1.2 PDs per AC cycle.

By describing the surface charge density from a discharge, $\sigma_{\mathrm{PD} i}$, as a continuous function in equation (7) the surface of the void does not need to be artificially discretized, a procedure which is required in existing work where the distribution of surface charge from a PD is assumed to be uniform in a given area [11]. The assumption of a uniform surface charge distribution also creates numerical issues from the discontinuity in the gradient of the electric field, a problem not present with a continuous distribution.

PDs only occur in a single half cycle so the surface charge must decay between discharges, otherwise PD activity would rapidly cease. An exponential decay has been assumed so that between discharges

$$
\sigma(\theta, \phi, t)=\sigma\left(\theta, \phi, t_{\mathrm{PD} i}^{+}\right) \exp \left(-\frac{t-t_{\mathrm{PD} i}}{\tau}\right),
$$

where $t_{\mathrm{PD} i} \leq t<t_{\mathrm{PD} i+1}, \tau$ is an appropriate time decay constant, adjusted to fit the data. The assumption of an exponential decay of surface charge, and the electric field it creates, has been used previously by other researchers $[13,15,16]$.

\subsection{PD INCEPTION}

PDs are only observed in the negative half cycle of the applied field, so it is assumed that initial electrons for a discharge are generated by the spike. To model this, PDs are allowed to occur at any of $N_{\text {inc }}$ approximately evenly distributed points, referred to as inception points, on the spike tip. It is important to note that $N_{\text {inc }}$ must be sufficiently high to ensure that the electric field is approximately uniform in the vicinity of each inception point. In the arrangement considered model behavior did not change for $N_{\text {inc }}>100$. The inception points were defined using a Fibonacci grid algorithm to ensure an even distribution of points in space.

The field condition requires that the electric field normal to the surface is higher than an inception field $E_{\text {inc }}$. Explicitly for the field condition to met at the $i$ th inception point at time $t$

$$
\left|E_{\text {spike } i}(t)\right|>E_{\text {inc }}
$$

where

$$
E_{\text {spike } i}(t)=\vec{E}\left(\vec{x}_{\text {inc } i}, t\right) \cdot \vec{n}_{\text {spike }}\left(\vec{x}_{\text {inc } i}\right),
$$

$\vec{x}_{\text {inc } i}$ is the spatial coordinates of the $i$ th inception point and $\vec{n}_{\text {spike }}$ is an outward facing normal vector at the spike surface.

The mechanism of electron generation from the metallic spike is a complicated process and it is beyond the scope of this model to fully capture all physical phenomena. Previously in the literature PD activity from a needle-plane configuration in air was accurately modeled by assuming that the electron generation rate, $\dot{N}_{e}$, was proportional to $\left(|\vec{E}|-E_{\text {inc }}\right)^{3}$ in the negative half cycle and zero otherwise [13]. It is reasonable to assume that electron generation from the metallic spike will obey a similar scaling and it was found that a good fit with the 
experiment could be achieved by assuming that electron generation scaled with the cube of the field strength in the negative half cycle. Formally the electron generation rate at the $i$ th inception point, $\dot{N}_{e i}(t)$, is then

$$
\dot{N}_{e i}(t)=\left\{\begin{array}{cl}
\frac{c_{e}}{N_{\text {inc }}}\left|E_{\text {spike } i}(t)\right|^{3} & \text { if } E_{\text {spike } i}(t)<0 \\
0 & \text { if } E_{\text {spike } i}(t) \geq 0
\end{array}\right.
$$

where $\mathrm{c}_{e}$ is an adjustable parameter set such that the simulation results more closely resemble the experimental data. The free electron condition depends on the electron generation rate, it will be assumed that an electron is available at the $i$ th inception point at time $t$ if

$$
\dot{N}_{e i}(t) \Delta t>R \text {, }
$$

where $\Delta t$ is the time step used in the model and $R$ is a random number uniformly distributed between 0 and 1 . The model results are independent of time step choice, as long as it is sufficiently small compared to the length of the AC cycle; in this work the time step, $\Delta t$, is set to $1 \times 10^{-5} \mathrm{~s}$.

If both the field condition, equation (10), and the free electron condition, equation (12), are met then a PD will occur at that inception point. Both conditions must be considered at each point in turn so the ordering of the points is randomly permuted at each time step to remove bias. At a discharge event the surface charge density at the void surface will be changed, equation (6), such that the electric field at the inception point that PD is occurring at is reduced to $E_{\text {ext }}$. Therefore, for the $j$ th PD occurring at time $t_{\mathrm{PD} j}$, from the $i$ th inception point

$$
\left|E_{\text {spike } i}\left(t_{\mathrm{PD} j}^{+}\right)\right|=E_{\text {ext }} \text {. }
$$

\subsection{APPARENT CHARGE}

The apparent charge, $q_{\mathrm{PD}}^{\prime}$, from a given PD can be calculated by numerically integrating the change in surface charge density due to PD across the measuring electrode [11]. The measuring electrode used in the experiment was phase two, so to determine the apparent charge from PDs in the model the change in surface charge density is integrated across a surface $S$ comprising of phase two and the spike. The apparent charge of the $i$ th $\mathrm{PD}, q_{\mathrm{PD} i}^{\prime}$, is then

$$
\begin{aligned}
& q_{\mathrm{PD} i}^{\prime} \\
& =\int_{S}\left(\vec{D}\left(\vec{x}_{S}, t_{\mathrm{PD} i}^{+}\right)-\vec{D}\left(\vec{x}_{S}, t_{\mathrm{PD} i}^{-}\right)\right) \cdot \vec{n}_{\mathrm{S}}\left(\vec{x}_{S}\right) d S
\end{aligned}
$$

where $\vec{x}_{S}$ are spatial coordinates on $S, \vec{D}$ is the electric displacement field and $\vec{n}_{\mathrm{S}}$ is an outward facing normal vector on $S$.

\subsection{ADJUSTABLE PARAMETERS}

The governing equations and assumptions of the model have now been introduced, it remains to choose the adjustable, often referred to as free, parameters appropriately so that the model results match those of the experiment. The model has 3 free parameters: $R_{\mathrm{void}}, \tau$ and $\mathrm{c}_{e}$, which is comparable with existing PD models in the literature $[3,6,15]$. The values of all parameters used in the simulation are given in Table 1.

$E_{\text {inc }}$ and $E_{\text {ext }}$ are not free parameters because they are dependent on another free parameter, the void radius. This follows from the fact that PDs occur relative infrequently and that the surface charge decays between PD. Therefore when the time between PDs is sufficiently large, $\vec{E} \approx \vec{E}_{0}$, where $\vec{E}_{0}$ is the applied field due to the applied voltage, equation (2). The highest magnitude PD possible in the system will then occur when the applied field is at its maximum value in the AC cycle. Therefore for a given void radius, $E_{\text {ext }}$ can be found such that the highest magnitude PD that can occur in the system matches the highest magnitude PD in the experimental data. The void radius will be adjusted such that this value of $E_{\text {ext }}$ is physically

\begin{tabular}{|c|c|c|}
\hline Parameter Group & Parameter & Value \\
\hline Electric Field & $\begin{array}{l}E_{\text {inc }} \\
E_{\text {ext }} \\
\tau^{*} \\
\varepsilon_{r \text { paper }} \\
\varepsilon_{r \text { oil }} \\
\varepsilon_{r \text { void }} \\
\end{array}$ & $\begin{array}{l}6.7 \mathrm{kV} \mathrm{mm}^{-1} \\
3.3 \mathrm{kV} \mathrm{mm}^{-1} \\
15 \mathrm{~ms} \\
3.6 \\
2.7 \\
1 \\
\end{array}$ \\
\hline Electron Generation & $c_{e}^{*}$ & $0.72 \mathrm{~mm}^{3} \mathrm{kV}^{-3}$ \\
\hline Geometry & $\begin{array}{l}R_{\text {joint }} \\
R_{\text {phase }} \\
R_{\text {paper }} \\
D_{\text {phase }} \\
L_{\text {spike }} \\
R_{\text {spike top }} \\
R_{\text {spike bottom }} \\
R_{\text {void }} *\end{array}$ & $\begin{array}{l}70 \mathrm{~mm} \\
8 \mathrm{~mm} \\
28 \mathrm{~mm} \\
35 \mathrm{~mm} \\
14 \mathrm{~mm} \\
0.25 \mathrm{~mm} \\
2 \mathrm{~mm} \\
2 \mathrm{~mm}\end{array}$ \\
\hline
\end{tabular}
sensible. Once $E_{\text {ext }}$ has been determined $E_{\text {inc }}$ can be found using the fact that the lowest magnitude PDs will occur when $\left|E_{\text {spike }}\right| \approx E_{\text {inc }}$.

Table 1. Parameter values.

\section{RESULTS}

Two different voltage boundary conditions were used in the model, equation (2), and the voltage waveform recorded in the experiment. The voltage waveform of the experiment represents a slight deviation from a typical sine function, as shown in Figure 6. Since data set 1 was collected over 990 AC cycles as opposed to data set 2 , which was collected over only 320 cycles, the model was fitted to data set 1 . It should be noted that the model describes both data sets well with a single choice of parameters due to the similarity between the data sets. Reasonable agreement was seen between PRPD patterns of experiment and simulation with both sinusoidal and measured waveform boundary conditions, with a comparable number of PDs per cycle and PD magnitudes. Discrepancies were observed in the shape of the "rabbit ear" PRPD pattern, with a more pronounced curvature in the model's "ears" compared to the experiment, as shown in Figure 7. There are also disparities in the phase angle range between experiment and simulation, this was primarily due to a few outlying data points in the simulation. However, the model was in close agreement with the data in the number of PDs per cycle and the phase magnitude range. A full summary of results is given in Tables 2 , 3 and 4 . 


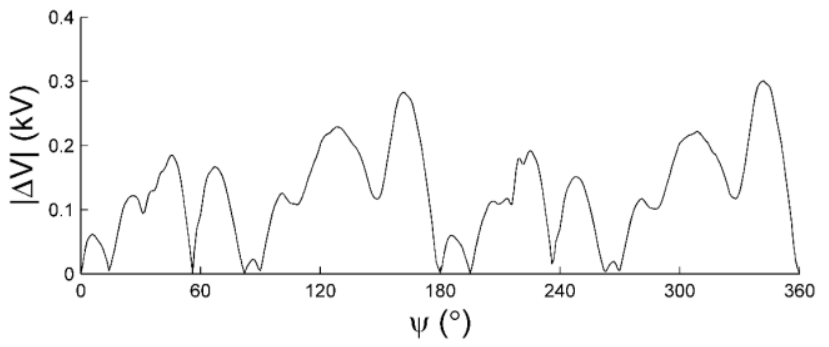

Figure 6. Figure showing the absolute difference between the average voltage waveform measured in the experiment and a sinusoidal waveform over an AC cycle.

Table 2. PD Experiment Results

\begin{tabular}{|c|c|c|}
\hline & Data Set 1 & Data Set 2 \\
\hline PDs per cycle & 1.2 & 1.2 \\
\hline Maximum $q_{\mathrm{PD}}^{\prime}(\mathrm{nC})$ & 2.4 & 2 \\
\hline Minimum $q_{\mathrm{PD}}^{\prime}(\mathrm{nC})$ & 0.55 & 0.55 \\
\hline Mean $q_{\mathrm{PD}}^{\prime}(\mathrm{nC})$ & 1.2 & 1.2 \\
\hline Phase Angle Range $\left({ }^{\circ}\right)$ & 85 & 79 \\
\hline
\end{tabular}

Table 3. PD Simulation Results 1

\begin{tabular}{l|l|l}
\hline & Sine Wave AC Cycle & $\begin{array}{c}\text { Measured Voltage } \\
\text { Waveform }\end{array}$ \\
\hline PDs per cycle & 1.3 & 1.3 \\
Maximum $q_{\mathrm{PD}}^{\prime}(\mathrm{nC})$ & 2.1 & 2.2 \\
Minimum $q_{\mathrm{PD}}^{\prime}(\mathrm{nC})$ & 0.56 & 0.55 \\
Mean $q_{\mathrm{PD}}^{\prime}(\mathrm{nC})$ & 1.3 & 1.2 \\
Phase Angle Range $\left(^{\circ}\right)$ & 98 & 97 \\
\hline
\end{tabular}

Table 4. PD Simulation Results 2

\begin{tabular}{l|l}
\hline & \multicolumn{2}{c}{ Phase 2 Energized Only } \\
\hline PDs per cycle & 1.0 \\
Maximum $q_{\mathrm{PD}}^{\prime}(\mathrm{nC})$ & 1.6 \\
Minimum $q_{\mathrm{PD}}^{\prime}(\mathrm{nC})$ & 0.57 \\
Mean $q_{\mathrm{PD}}^{\prime}(\mathrm{nC})$ & 1.0 \\
Phase Angle Range $\left(^{\circ}\right)$ & 99 \\
\hline
\end{tabular}

In addition to modeling the experimental data, the model is used to investigate an alternative testing environment. In many commercial tests, three-phase cable experiments are conducted with only a single energized phase. As the defect is located near phase 2 , the model was run with only phase 2 energized, with all other phases set to ground, the results are shown in Figure $7 \mathrm{c}$. Using only a single energized phase resulted in fewer PDs and a significantly different PRPD pattern which was phase shifted to the right by approximately $15^{\circ}$. There are two opposing factors that create this phase-shift. The PRPD pattern is shifted to the right because the electric field only depends on the phase angle of phase 2, when the cable is fully energized the other phases impact the electric field. When only phase two energized the electric field waveform is phase shifted to the right and has a lower magnitude, which results in the inception field being reached later in the AC cycle shifting the PRPD pattern to the right. The PRPD pattern is shifted to the left because the lower electric field results in fewer PDs, as the electron generation rate is lower and it is above the inception field for less time, which leads to less charge at the void surface opposing the electric field, so the electric field reaches the inception value at lower phase angles, shifting the PRPD pattern to the left. For the conditions in the simulation the net result is that the pattern is phase shifted to the right by $15^{\circ}$.

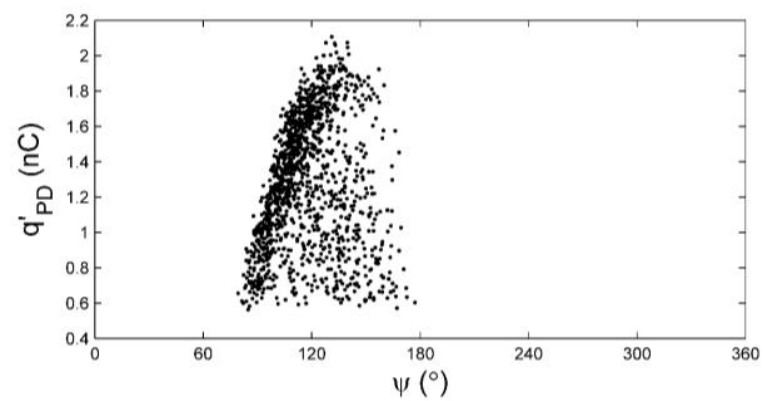

(a)

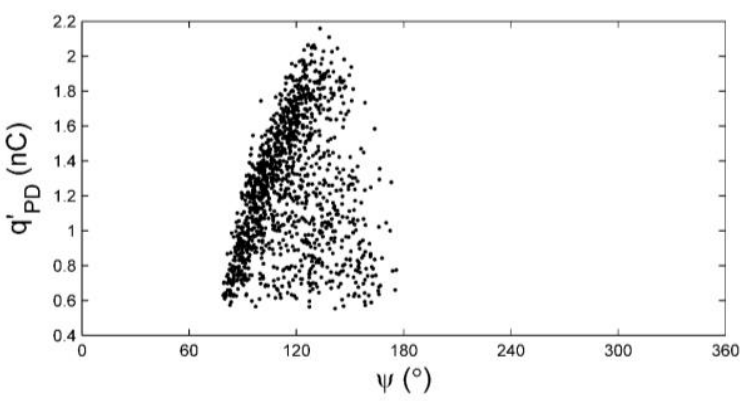

(b)

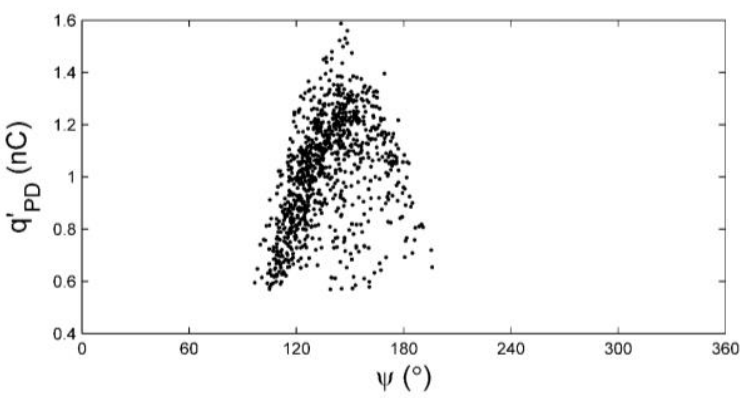

(c)

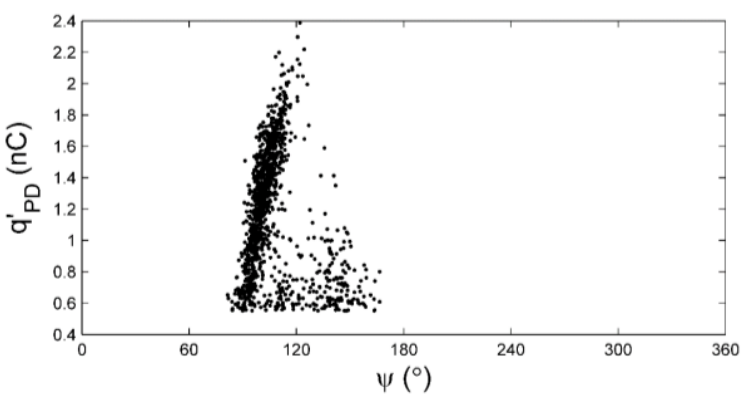

(d)

Figure 7. PRPD patterns for: (a) model with sinusoidal waveform boundary conditions, (b) model with measured voltage waveform boundary conditions, (c) model with phase 2 energized only and (d) PD data from spike defect (data set 1). All PRPD data is from 990 AC cycles. 


\section{DISCUSSION}

The level of agreement between experiment and simulation justifies the assumptions and simplifications made in the model. It is an encouraging result that a PD model with relatively few free parameters was able to reproduce data from a three phase cable experiment at rated conditions. The model represents an improvement over an earlier model by the authors of the same experiment that required a larger number of free parameters to fit the data, including extra stochastic terms, and did not consider the defect geometry [17]. One of the main contributions of this model is that it is applicable to systems with a range of applied fields, and where PD can occur at different locations. Furthermore it describes PDs using continuous surface charge density distributions, which offer advantages in terms of solution accuracy and do not require the surface to be artificially discretized.

The free parameters used to describe the model are physically sensible, and have implications for the PD system. Firstly, the void is relatively large with a radius of $2 \mathrm{~mm}$, suggesting that the presence of the spike has caused significant damage to the Crêpe paper to trap this volume of air close to phase 2. It is also interesting that an empirical fit used for the electron generation rate of a needle-plane system could be successfully applied to a metallic spike under different conditions. It is likely that a better fit to the experimental data could have been achieved if more parameters were used. However, due to the high level of uncertainty in the experimental data it was decided to obtain a reasonable fit using fewer free parameters and a more general model. An issue with introducing more free parameters is that it increasingly reduces the model to a curve-fitting exercise, which was not the aim of the research.

Another interesting result from the study is the dependence of PD on the applied field. Typically in PD modeling a perfectly sinusoidal applied field is assumed. The model results reinforce the validity this assumption, at least for the case considered, with the measured waveform giving similar results to the sinusoidal waveform. There are still detectable differences between the sinusoidal and measured waveform boundary conditions which were identified by comparing a large number simulation runs. The most obvious of these is that measured waveform simulation has a phase angle distribution shifted to the left by an average of $3^{\circ}$ compared to the phase angle distribution of the sinusoidal simulation. This was due to the different shape of the waveform, leading the inception field to be reached at lower phase angles. The model also showed significant differences between PRPD patterns for a single energized phase compared to a fully energized cable. The results from the simulation suggest that the applied voltage input should be closely monitored when testing PD activity in high voltage plant, and that PD measurements are taken under rated volts with the same energization as operating conditions.

The experimental results show that PD activity is heavily dependent on temperature, with the results implying that PD testing of PILC cable sections for spike defects should be conducted at higher temperatures as it leads to a dramatic reduction in the background $\mathrm{PD}$ from cable terminations. A possible reason for this is that the PD from the cable terminations is caused by numerous gas filled voids formed in the heat shrink materials used to create the termination. As these voids are fixed in size, increasing the temperature will lead to an increase in pressure, subsequently increasing the inception and extinction electric fields, through equations (4) and (16) in [18]. This explains the reduction in PD magnitude and frequency from cable terminations.

Despite this success, there are discrepancies between the model and experiment. One of the possible explanations for this is that spike geometry used in the model represents a simplification of the more complex geometry present in the experiment, which has implications on the apparent charge magnitude as it is calculated through an integral whose domain includes the spike surface. An alternative spike geometry would also influence the form of the electric field, which has been already demonstrated to impact PD activity. Another simplification made in the model is that the surface charge distribution is independent of the location on the spike the discharges initiate from. Furthermore, variations in $c_{e}$ and $\tau$ may occur over the AC cycle. There are also uncertainties present in the measuring process, with a high level of background noise, discrimination of spike PD data is nontrivial, even at higher temperatures, and data assumed to originate from the spike may be contaminated with PD from the terminations. These problems are compounded by cross-talk between phases and attenuation of the PD pulse, leading to a greater uncertainty in the measurement of the apparent charge. This contrasts with more controlled PD experiments between parallel plates, where cross-talk and attenuation is negligible as the void is typically close to the measuring electrode and surrounded by a homogeneous dielectric material.

Finally, it should also be noted that the model presented is only preliminary step in simulating on-site data, before this can be attempted there are several obstacles that must be overcome. An obvious difficulty is that the model required a known defect type, location and size, all of which may be unknowns in PD data taken on-site. There is also only a single known source of PD present in this experiment, whereas in the operational plant there can be many sources which may also be interacting.

\section{CONCLUSION}

In this work a PD model has been introduced that reproduces PD data from a three-phase cable experiment. The model requires only 3 free parameters, and was able to give reasonable agreement with experimental data, with good agreement in PDs per cycle, mean PD magnitude and phase angle range. The model extends previous work by considering discharge inception at multiple points in the defect, and by modeling surface charge density through continuous functions. It was concluded that PD testing of three-phase cable joints should be performed at higher temperatures with all three-phases energised to be able to reduce PD from cable terminations and to reproduce the electric field present in the joint at rated conditions. The limitations of the model were discussed, including that the information known in an experiment may not be available in the field. This work is a preliminary step towards simulating on-site data; improvements in experimental and modeling techniques are required before models can be developed to fully investigate on-site PD measurements. 


\section{ACKNOWLEDGMENTS}

This work was supported by an EPSRC Doctoral Training Centre grant (EP/G03690X/1). The authors are grateful for the use of facilities at the Tony Davies High Voltage Laboratory, University of Southampton.

\section{REFERENCES}

[1] R. J. Van Brunt, "Physics and chemistry of partial discharges and corona. Recent advances and future challenges", IEEE Trans. Dielectr. Electr. Insul., Vol. 1, pp. 761-784, 1994.

[2] S. A. Boggs, "Partial discharge: overview and signal generation", IEEE Electr. Insul. Mag., Vol. 6, No. 4, pp. 33-39, 2014.

[3] H. A. Illias, G. Chen and P. L. Lewin, "Partial discharge behavior within a spherical cavity in a solid dielectric material as a function of frequency and amplitude of the applied voltage", IEEE Trans. Dielectr. Electr. Insul., Vol. 18, pp. 432-443, 2011.

[4] K. Wu, C. Pan, Y. Meng and Y. Cheng, "Dynamics behavior of surface charge distribution during partial discharge sequences", IEEE Trans. Dielectr. Electr. Insul., Vol. 20, pp. 612-619, 2013.

[5] C. Pan, Y. Meng, K. Wu, Z. Han, K. Qin and Y. Cheng, "Simulation of partial discharge sequences using fluid equations", J. Phys. D: Appl. Phys., Vol. 44, 255201, 2011.

[6] C. Forssen and H. Edin, "Partial discharges in a cavity at variable applied frequency part 2: Measurements and modeling”, IEEE Trans. Dielectr. Electr. Insul., Vol. 15, pp. 1610-1616, 2008.

[7] L. Niemeyer, "A generalized approach to partial discharge modeling", IEEE Trans. Dielectr. Electr. Insul., Vol. 2, pp. 510-528, 1995.

[8] L. Hao, P. L. Lewin, J. A. Hunter, D. J. Swaffield, A. Contin, C. Walton and M. Michel, "Discrimination of multiple pd sources using wavelet decomposition and principal component analysis", IEEE Trans. Dielectr. Electr. Insul., Vol. 18, pp. 1702-1711, 2011.

[9] J. A. Hunter, An investigation into partial discharge activity within threephase belted cables, Ph.D. thesis, University of Southampton, UK, 2013.

[10] J. A. Hunter, L. Hao, P. L. Lewin, C. Walton and M. Michel, "Partial discharge diagnostics of defective medium voltage three-phase pilc cables", IEEE Int'l. Sympos. Electr. Insul., San Juan, PR, pp. 371-375, 2012.

[11] H. A. Illias, G. Chen and P. L. Lewin, "The influence of spherical cavity surface charge distribution on the sequence of partial discharge events", J. Phys. D: Appl. Phys., Vol. 44, 245202, 2011.

[12] J. A. Hunter, L. Hao, D. J. Swaffield, P. L. Lewin, N. Cornish, C. Walton and M. Michel, "Partial discharge in medium voltage three-phase cables", IEEE Int'l. Symp. Electr. Insul., San Diego, CA, USA, paper 131, 2010.

[13] C. Heitz, "A generalized model for partial discharge processes based on a stochastic process approach", J. Phys. D: Appl. Phys., Vol. 32, pp. 1012$1023,1999$.

[14] R. Ono and T. Oda, "Optical diagnosis of pulsed streamer discharge under atmospheric pressure”, Int'l. J. Plasma Environ. Sci. Technol., Vol. 1, pp. 123-129, 2007

[15] A. Cavallini and G. C. Montanari, "Effect of supply voltage frequency on testing of insulation system”, IEEE Trans. Dielectr. Electr. Insul., Vol. 13, pp. 111-121, 2006.

[16] G. Chen and Z. Xu, "Charge trapping and detrapping in polymeric materials", J. Appl. Phys., Vol. 106, 123707, 2009.

[17] G. Callender, J. A. Hunter, P. Rapisarda and P. L. Lewin, "Physical models for field based partial discharge measurements", IEEE Electr. Insul. Conf., Seattle, WA USA, pp. 519-522, 2015.

[18] F. Gutfleisch and L. Niemeyer, "Measurement and simulation of PD in epoxy voids", IEEE Trans. Dielectr. Electr. Insul., Vol. 2, pp. 729-743, 1995.

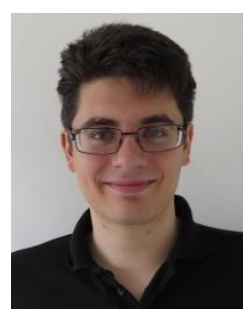

George Callender was born in Basildon, UK in 1991. He received M.Sc. (Hons.) in Natural Sciences (Maths and Physics) from the University of Durham, UK in 2013. He is currently a Ph.D. student at the University of Southampton. His research interests include partial discharge modeling and partial discharge source discrimination.

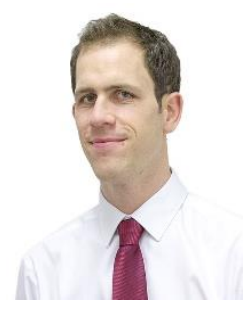

Jack A. Hunter was born in Reading, UK in 1987. He received the B.Eng. (Hons) in electrical engineering from the University of Southampton, UK in 2009. He then worked as a post-graduate researcher at the Tony Davies High Voltage Laboratory. Currently he is working at Mott MacDonald focusing on HV cable projects. Recent projects involve UK OFTO transfer due diligence, Technical Advisor role to an existing OFTO operator, $\mathrm{NG}$ interconnector tender standard advisor and project managing a NR MV reinforcement project. He is a member of the Cigre Task Force B1.58.

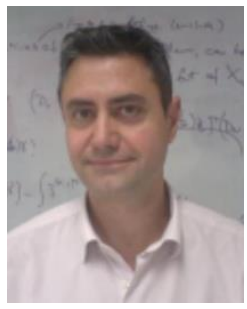

Paolo Rapisarda received a Laurea (M.Sc.) degree in Computer Science at the University of Udine, Italy; and a Ph.D. in Mathematical Systems and Control Theory at the University of Groningen, The Netherlands, working under the supervision of Jan C. Willems and Harry L. Trentelman. He has worked as a Lecturer at the Department of Electrical, Computer and Electronics Engineering of the University of Trieste, Italy; and at the Department of Mathematics of the University of Maastricht, The Netherlands. Currently he is Senior Lecturer at the Information: Signals, Images, Systems group of the School of Electronics and Computer Science of the University of Southampton, UK.

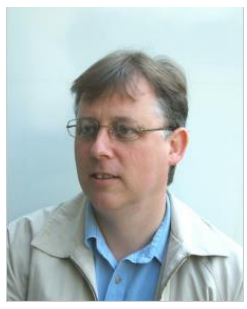

Paul L. Lewin (M'05-SM'08) was born in Ilford, Essex in 1964. He received the B.Sc. (Hons) and Ph.D. degrees in electrical engineering from the University of Southampton, UK in 1986 and 1994, respectively. He joined the academic staff of the University in 1989 and is the Professor of Electrical Power Engineering in the School of Electronics and Computer Science, where he is also Director of the Tony Davies High Voltage Laboratory. His research interests are within the generic areas of applied signal processing and control. Within high voltage engineering this includes condition monitoring of $\mathrm{HV}$ cables and plant, surface charge measurement, $\mathrm{HV}$ insulation/dielectric materials and applied signal processing. Since 1996 he has received funding and grants in excess of $£ 14 \mathrm{M}$, supervised 23 graduate students to successful completion of their doctoral theses and published over 270 refereed conference and journal papers in these research areas. He is a Chartered Engineer, a Fellow of the IET, president of the IEEE Dielectrics and Electrical Insulation Society as well as an Associate Editor of IEEE Transactions on Dielectrics and Electrical Insulation. 